\title{
NUMERICAL SIMULATION OF DENGUE FEVER (SIR MODEL) USING DIFFERENTIAL TRANSFORM METHOD, MULTI-STEP DIFFERENTIAL TRANSFORM METHOD AND RK4 METHOD
}

\author{
Gurpreet Singh Tuteja \\ Zakir Husain Delhi College, J. L. Nehru Marg, New Delhi-110002, India
}

\begin{abstract}
This study investigates the application of the differential transformation method(DTM), multi-step differential transform method(MsDTM) with step-size and RK4 method (Mathematica) for finding the numerical solution of the SIR model of dengue fever in epidemiology. This model is a system of non-linear ordinary differential equations that have no analytic solution. Both the methods DTM and MsDTM are applied directly without any linearization, perturbation or discretization in the model equations to obtain semi-analytic solutions. The accuracy of the MSDTM is excellent and comparable to the RK4 method of Mathematica.
\end{abstract}

Keyword: SIR model, Differential Transformation Method (DTM), Multi-step Differential Transform Method (MsDTM), RK4

MSC2010: 91A40

\begin{abstract}
Introduction
Dengue is a mosquito-borne flavivirus found mainly in an urban and semi-urban area in tropical and subtropical regions of the world. Aedes mosquitoes transmit disease through day-biting. It is the fastest spreading viral disease transmitted by vectors and is now endemic in over 100 countries, resulting in $40 \%$ of the world's population living in a dengue-risk region [7]. Between 1990 and 2013, the incidence of dengue increased dramatically, with the number of cases more than doubling per decade, from 8.3 million obvious cases in 1990 to 58.4 million apparent cases in 2013 [29]. Over the last two decades, the number of dengue cases registered in the records of WHO has raised more than 8 folds, from 505,430 cases in 2000 to over 2.4 million in 2010 and 4.2 million in 2019. Reported deaths also rose from 960 to 4032 between the years 2000 and 2015 [8]. In epidemiology, the spread of diseases in the population is studied through the mathematical formulation of a model for contagious diseases, investigation of related parameters, the sensitivity of
\end{abstract}

the model by simulating the parameters and present numerical simulations. Such modelling not only helps to study the patterns of the spread of disease but also the possibility to control it optimally. Some of the examples of such contagious diseases are COVID19, Dengue, E-bola, Measles, Rubella, Chicken-pox, HIV/AIDS, Syphilis and others.

To analyse the spread of infectious diseases, various epidemiological models, including the compartment model [6], have been developed. This compartment model, based on the epidemiological status of the population, is divided into three distinct mutually exclusive compartments: susceptible $S(t)$, infectious $I(t)$ and recovered $R(t)$ at any time $t$. Several types of compartmental models [14-16,19-21] were studied, such as SI (susceptible-infected), SIS (susceptibleinfected-susceptible), SIR (susceptible-infectedrecovered), SIRS (susceptible-infected-recoveredsusceptible), and SEIRS (susceptible-exposedinfected-recovered). The movement of the population from one compartment to another depends on the transmission rate [11]. Here, in this SIR model, two separate but dependent sets of non-linear differential equations related to human and vector (mosquito) population are considered [9]. The purpose of this paper is to find the semi-analytical solution of the dengue fever (SIR) model with limited immunity and compare the numerical solutions obtained by using different methods: differential transform method (DTM), multi-step differential transform (with different step-size) method (MsDTM) and RungeKutta Method (RK4) using Wolfram Mathematica. The paper is organized in the following sections: In section 2, the SIR model for dengue fever with model parameters is briefly described. Section 3, discusses the existence, uniqueness and positivity of the solution. Section 4, briefly introduces the theoretical and implementation of DTM, MsDTM. Section 5, contains numerical solutions and a brief discussion. Finally, section 6 has a conclusion.

\section{Formulation of SIR model for Dengue Fever}

In this mathematical model, we assume that host and vector populations have a constant size. The birth and death rates for human $\mu_{h}$ and vector $\mu_{v}$ are constants. The human population is divided into three mutually 
exclusive compartments viz. susceptible $S_{h}$, infected $I_{h}$, and recovered $R_{h}$, while vector population due to short life span is divided into two classes only: susceptible $S_{v}$ and infected $I_{v}$. There is no recovered compartment for vectors, as with the death of a vector, the period of infection also ends. The model of human population and vector is given in Fig. 1 which is given as a set of non-linear differential equations [31]:

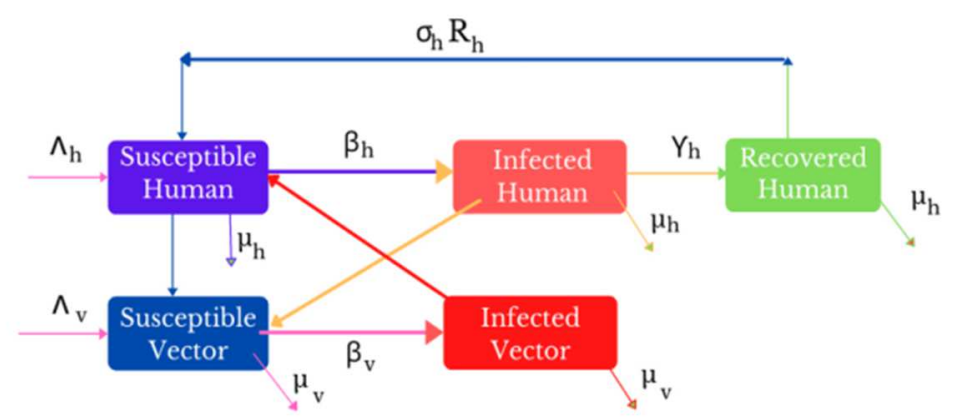

Fig. 1: Dengue Fever Model

$$
\begin{aligned}
\frac{d S_{h}}{d t} & =\Lambda_{h} N_{h}-\frac{\beta_{h} b}{N_{h}} S_{h}(t) I_{v}(t)-\mu_{h} S_{h}(t)+\sigma_{h} R_{h}(t) \\
\frac{d I_{h}}{d t} & =\frac{\beta_{h} b}{N_{h}} S_{h}(t) I_{v}(t)-\left(\mu_{h}+\gamma_{h}\right) I_{h}(t) \\
\frac{d R_{h}}{d t} & =\gamma_{h} I_{h}(t)-\mu_{h} R_{h}(t)-\sigma_{h} R_{h}(t) \\
\frac{d S_{v}}{d t} & =\Lambda_{v} N_{v}-\frac{\beta_{v} b}{N_{h}} S_{v}(t) I_{h}(t)-\mu_{v} S_{v}(t) \\
\frac{d I_{v}}{d t} & =\frac{\beta_{v} b}{N_{h}} S_{v}(t) I_{h}(t)-\mu_{v} I_{v}(t) . \\
\text { Where } & N_{h}(t)=S_{h}(t)+I_{h}(t)+R_{h}(t), \text { and } N_{v}(t)=S_{v}(t)+I_{v}(t),
\end{aligned}
$$$$
S_{h}(0)=S_{h 0} \geq 0, I_{h}(0)=I_{h 0} \geq 0, R_{h}(0)=R_{h 0} \geq 0, S_{v}(0)=S_{v 0} \geq 0, I_{v}(0)=I_{v 0} \geq 0 \text {, }
$$

The parameters $\Lambda_{h} N_{h}$ is a change in the total human population (the rate of recruitment of human or birth rate including migration is $\Lambda_{h}$ ). The probability of a susceptible individual being infected with the dengue virus is $\beta_{h} b I_{v} / N_{h}$, where $\beta_{h}$ is the probability of getting the infection from an infected vector to a susceptible human; $b$ denotes a vector's average bites. $\mu_{h} S_{h}$ represents the number of deaths among susceptible human population and $\mu_{h} I_{h}$ is the number of deaths in the infected human population while those infected and recovered from the infection are represented by $\gamma_{h} I_{h} . R_{h}$ is the total human population that has recovered from the infection but doesn't gain immunity forever. $\sigma_{h} R_{h}$ is the population that recovered and is immune for a limited period and then joins back the susceptible after a certain period

$$
\begin{aligned}
\frac{d S_{h}}{d t} & =\Lambda_{h}-\xi_{1} S_{h}(t) I_{v}(t)-\mu_{h} S_{h}(t)+\sigma_{h} R_{h}(t), \\
\frac{d I_{h}}{d t} & =\xi_{1} S_{h}(t) I_{v}(t)-\left(\mu_{h}+\gamma_{h}\right) I_{h}(t), \\
\frac{d R_{h}}{d t} & =\gamma_{h} I_{h}(t)-\mu_{h} R_{h}(t)-\sigma_{h} R_{h}(t), \\
\frac{d S_{v}}{d t} & =\Lambda_{v}-\xi_{2} S_{v}(t) I_{h}(t)-\mu_{v} S_{v}(t), \\
\frac{d I_{v}}{d t}= & \xi_{2} S_{v}(t) I_{h}(t)-\mu_{v} I_{v}(t) . \\
& \text { Where, } \quad \xi_{1}=\frac{\beta_{h} b N_{v}}{N_{h}} \text { and } \xi_{2}=\beta_{v} b \text { and } \xi_{1}, \xi_{2}>0, \text { as } \mathrm{b}>0
\end{aligned}
$$


The differential transformation method is one of the well-known techniques to solve both linear, nonlinear differential equations and partial differential equations. It was first introduced by Zhou [34] for solving linear and non-linear initial value problems in electrical circuit analysis. Subsequently, several authors have applied the differential transformation method (DTM) and further multi-step differential transform method (MsDTM) to solve systems of nonlinear differential equations that describe dynamical systems [30], biomathematics models [18] and epidemic models [1-3,14,23,26,33]. Several variants have been suggested for differential transformation method like modified DTM [5], reduced DTM $[17,22,28]$ and partitioned DTM [4]. These methods are used to write a semi-analytical solution of the model which depends on the Taylor series [27]. Studies have compared the DTM and Multi-Step DTM method and reported that solutions match but only for a small value of the independent variable [24].

\section{Existence, Uniqueness and Positivity of Solution}

We will use the Lipchitz condition to verify the existence and uniqueness of solution [10] for the model equations (7) - (11):

$$
\begin{aligned}
& E_{1}=\Lambda_{h}-\xi_{1} S_{h}(t) I_{v}(t)-\mu_{h} S_{h}(t)+\sigma_{h} R_{h}(t), \\
& E_{2}=\xi_{1} S_{h}(t) I_{v}(t)-\left(\mu_{h}+\gamma_{h}\right) I_{h}(t), \\
& E_{3}=\gamma_{h} I_{h}(t)-\left(\sigma_{h}+\mu_{h}\right) R_{h}(t), \\
& E_{4}=\Lambda_{v}-\xi_{2} S_{v}(t) I_{h}(t)-\mu_{v} S_{v}(t), \\
& E_{5}=\xi_{2} S_{v}(t) I_{h}(t)-\mu_{v} I_{v}(t) .
\end{aligned}
$$

Let $\mathrm{B}$ denote the region, $\left|t-t_{0}\right| \leq \delta,|| x-x_{0}|| \leq$ $\alpha$, where $x=\left(x_{1}, x_{2}, \ldots x_{n}\right), x_{0}=\left(x_{10}, x_{20}, \ldots x_{n 0}\right)$ also suppose that $a(t, x)$ satisfies the Lipschitz condition:

$$
\left\|a\left(t, x_{1}\right)-a\left(t, x_{2}\right)\right\| \leq k\left\|x_{1}-x_{2}\right\|
$$

Whenever the pairs $\left(t, x_{1}\right),\left(t, x_{2}\right)$ belong to $B$ where $\mathrm{k}$ is a positive constant, then there is a positive constant $\delta \geq 0$, such that there exists a unique and continuous vector solution $x(t)$ of the system in the interval $\mid t$ $t_{0} \mid<\delta$. The condition is satisfied by the requirement that $\frac{\partial a_{i}}{\partial x_{j}}, i, j=1,2,3, \ldots n$, be continuous and bounded in B. Considering the model equation (7)-(11), we are interested in the region $0 \leq \alpha \leq \mathrm{R}$ [26].

Let $\mathrm{B}$ denote the region $0 \leq \alpha \leq \mathrm{R}$, then equations (7) - (11) will have a unique solution if $\frac{\partial a_{i}}{\partial x_{j}}, i, j=$ $1,2,3, . .5$ are continuous and bounded in $\mathrm{B}$. For $\mathrm{E}_{1}$ :

For $\mathrm{E}_{2}$ :

$$
\begin{gathered}
\left|\frac{\partial E_{1}}{\partial S_{h}}\right|=\left|-\xi_{1} I_{v}(t)-\mu_{h}\right|<\infty,\left|\frac{\partial E_{1}}{\partial I_{h}}\right|=0<\infty,\left|\frac{\partial E_{1}}{\partial R_{h}}\right|=\left|-\sigma_{h}\right|<\infty,\left|\frac{\partial E_{1}}{\partial S_{v}}\right|=0<\infty, \\
\left|\frac{\partial E_{1}}{\partial I_{v}}\right|=0<\infty .
\end{gathered}
$$

For $\mathrm{E}_{3}$ :

$$
\begin{aligned}
& \left|\frac{\partial E_{2}}{\partial S_{h}}\right|=\left|\xi_{1} I_{v}(t)\right|<\infty,\left|\frac{\partial E_{2}}{\partial I_{h}}\right|=\left|\mu_{h}+\gamma_{h}\right|<\infty,\left|\frac{\partial E_{2}}{\partial R_{h}}\right|=0<\infty,\left|\frac{\partial E_{2}}{\partial S_{v}}\right|=0<\infty, \\
& \left|\frac{\partial E_{2}}{\partial I_{v}}\right|=0<\infty \text {. }
\end{aligned}
$$

$$
\begin{gathered}
\left|\frac{\partial E_{3}}{\partial S_{h}}\right|=0<\infty,\left|\frac{\partial E_{3}}{\partial I_{h}}\right|=\left|\gamma_{h}\right|<\infty,\left|\frac{\partial E_{3}}{\partial R_{h}}\right|=\left|-\sigma_{h}-\mu_{h}\right|<\infty,\left|\frac{\partial E_{3}}{\partial S_{v}}\right|=0<\infty, \\
\left|\frac{\partial E_{3}}{\partial I_{v}}\right|=0<\infty .
\end{gathered}
$$

These partial derivatives exist, continuous and are bounded, similarly for $\mathrm{E}_{4}, \mathrm{E}_{5}$. Hence the model has a unique solution. The positivity of the solution can be shown easily [31].

\section{DTM and MsDTM}

The differential transformation of the $k^{\text {th }}$ derivative of $u(x)$ is defined as:

We obtain,

$$
U(k)=\frac{1}{k !}\left[\frac{d^{k} u(x)}{d x^{k}}\right]_{x_{0}} .
$$

$$
u(x)=\sum_{k=0}^{\infty} U(k)\left(x-x_{0}\right)^{k}
$$

is called the inverse differential transformation of $U(k)$. In real applications, the function $u(x)$ can be expressed as a finite series and equation (13) can be expressed as 


$$
u(x)=\sum_{k=0}^{n} U(k)\left(x-x_{0}\right)^{k} .
$$

Also, from (3.1) and (3.2), we have

$$
u(x)=\sum_{k=0}^{n}\left(x-x_{0}\right)^{k} \frac{1}{k !}\left[\frac{d^{k} u(x)}{d x^{k}}\right]_{x=x_{0}} .
$$

From (12)and (13), the following properties can be obtained.

(1) If $\mathrm{z}(\mathrm{x})=\mathrm{u}(\mathrm{x}) \pm \mathrm{v}(\mathrm{x})$, then $\mathrm{Z}(\mathrm{k})=\mathrm{U}(\mathrm{k}) \pm \mathrm{V}(\mathrm{k})$.

(2) If $\mathrm{z}(\mathrm{x})=\alpha \mathrm{u}(\mathrm{x})$, then $\mathrm{Z}(\mathrm{k})=\alpha \mathrm{U}(\mathrm{k})$.

(3) If $\mathrm{z}(\mathrm{x})=\mathrm{u}^{\prime}(\mathrm{x})$, then $\mathrm{Z}(\mathrm{k})=(\mathrm{k}+1) \mathrm{U}(\mathrm{k}+1)$.

(4) If $\mathrm{z}(\mathrm{x})=\mathrm{u}^{\prime \prime}(\mathrm{x})$, then $\mathrm{Z}(\mathrm{k})=(\mathrm{k}+1)(\mathrm{k}+2) \mathrm{U}(\mathrm{k}+2)$.

(5) If $\mathrm{z}(\mathrm{x})=\mathrm{u}^{(l)}(\mathrm{x})$, then $\mathrm{Z}(\mathrm{k})=(\mathrm{k}+1)(\mathrm{k}+2) \ldots(\mathrm{k}+\mathrm{l}) \mathrm{U}(\mathrm{k}+\mathrm{l})$.

(6) If $\mathrm{z}(\mathrm{x})=\mathrm{u}(\mathrm{x}) \mathrm{v}(\mathrm{x})$, then $\mathrm{Z}(\mathrm{k}) \sum_{l=0}^{k} \mathrm{U}(\mathrm{l}) \mathrm{V}(\mathrm{k}-\mathrm{l})$.

(7) If $\mathrm{z}(\mathrm{x})=\alpha x^{l}$, then $\mathrm{Z}(\mathrm{k})=\alpha \delta(\mathrm{k}-1)$, where Kronecker delta $\delta(\mathrm{k}-1)\left\{\begin{array}{l}1, \text { if } k=l \\ 0, \text { if } k \neq l\end{array}\right.$.

Using the fundamental operations of differential transformation method, let $S_{h}(k), I_{h}(k), R_{h}(k), S_{v}(k)$ and $I_{v}(k)$ denote the differential transformations of $S_{h}(t), I_{h}(t), R_{h}(t), S_{v}(t)$ and $I_{v}(t)$ respectively, the recurrence relation to each equation of the system $(7)-(11)$ is as follow:

$$
\begin{aligned}
S_{h}[k+1] & =\frac{1}{k+1}\left\{\Lambda_{h} \delta(k, 0)-\mu_{h} \mathrm{~S}_{\mathrm{h}}[k]-\xi_{1} \sum_{l=0}^{k} \mathrm{~S}_{\mathrm{h}}[l] \mathrm{I}_{\mathrm{v}}[k-l]+\sigma_{\mathrm{h}} \mathrm{R}_{\mathrm{h}}[k]\right\} \\
I_{h}[k+1] & =\frac{1}{k+1}\left\{\xi_{1} \sum_{l=0}^{k} \mathrm{~S}_{\mathrm{h}}[l] \mathrm{I}_{\mathrm{v}}[k-l]-\left(\gamma_{\mathrm{h}}+\mu_{h}\right) \mathrm{I}_{\mathrm{h}}[k],\right. \\
R_{h}[k+1] & =\frac{1}{k+1}\left\{\gamma_{h} I_{h}[k]-\left(\mu_{h}+\sigma_{h}\right) R_{h}[k]\right\} \\
\mathrm{S}_{\mathrm{v}}[\mathrm{k}+1] & =\frac{1}{k+1}\left\{\Lambda_{\mathrm{v}} \delta[k, 0]-\mu_{v} \mathrm{~S}_{\mathrm{v}}[k]-\xi_{2} \sum_{l=0}^{k} \mathrm{~S}_{\mathrm{v}}[l] I_{h}[k-l]\right\} \\
\mathrm{I}_{\mathrm{v}}[\mathrm{k}+1] & =\frac{1}{k+1}\left\{\xi_{2} \sum_{l=0}^{k} \mathrm{~Sv}_{l}[l] \mathrm{I}_{h}[k-l]-\mu_{v} \mathrm{I}_{\mathrm{v}}[k]\right\}
\end{aligned}
$$

Now, we consider the initial conditions from [31], $S_{h}(0)=.99, I_{h}(0)=0.01, R_{h}(0)=0, S_{v}(0)=.99, I_{v}(0)=0.01$. Substituting the initial values to solve $S_{h}(k+1), I_{h}(k+1), R_{h}(k+1), S_{v}(k+1)$ and $I_{v}(k+1)$ in (16)-(20), we get, $s_{h}(t)$, $i_{h}(t), r_{h}(t), s_{v}(t)$ and $i_{v}(t)$ respectively. Then the closed form of the solution of order $6(k=6)$, can be written as: $s_{h}(t)=\sum_{k=0}^{6} S_{h}(k) t^{k}=0.99-0.000989 t-0.00008751605 t^{2}+\left(4.8357688 \times 10^{-7}\right) t^{3}-$ $\left(1.4188247 \times 10^{-7}\right) t^{4}+\left(3.9939127 \times 10^{-9}\right) t^{5}-\left(1.5241315 \times 10^{-10}\right) t^{6}$, $i_{h}(t)=\sum_{k=0}^{6} I_{h}(k) t^{k}=0.01-0.000011 t+0.00009311605 t^{2}-\left(3.6062986 \times 10^{-6}\right) t^{3}+$ $\left(2.3992481 \times 10^{-7}\right) t^{4}-\left(8.9904544 \times 10^{-9}\right) t^{5}+\left(3.106649 \times 10^{-10}\right) t^{6}$,

$r_{h}(t)=\sum_{k=0}^{6} I_{h}(k) t^{k}=0.001 t-\left(5.6 \times 10^{-6}\right) t^{2}+\left(3.1227217 \times 10^{-6}\right) t^{3}-\left(9.8042336 \times 10^{-8}\right) t^{4}+$ $\left(4.9965416 \times 10^{-9}\right) t^{5}-\left(1.5825175 \times 10^{-10}\right) t^{6}$, $s_{v}(t)=\sum_{k=0}^{6} \mathrm{~S}_{\mathrm{v}}(\mathrm{k}) \mathrm{t}^{\mathrm{k}}=0.99-0.00188 t+0.000012369 t^{2}-\left(6.196514 \times 10^{-6}\right) t^{3}+(2.0586103 \times$ $\left.10^{-7}\right) t^{4}-\left(1.0315079 \times 10^{-8}\right) t^{5}+\left(3.5314596 \times 10^{-10}\right) t^{6}$, $i_{v}(t)=\sum_{k=0}^{6} \mathrm{I}_{\mathrm{v}}(\mathrm{k}) \mathrm{t}^{\mathrm{k}}=0.01+0.00188 t-0.000012369 t^{2}+\left(6.196514 \times 10^{-6}\right) t^{3}-(2.0586103 \times$ $\left.10^{-7}\right) t^{4}+\left(1.0315079 \times 10^{-8}\right) t^{5}-\left(3.5314596 \times 10^{-10}\right) t^{6}$,

In the Multistep DTM, the interval $[0, T]$ is divided into M subintervals $\left[t_{i-1}, t_{i}\right], i=1,2 \ldots, M$ of equal step size $h=T / M$ by using the nodes $t_{i}=i h$, with step-size $h$. First, we apply the DTM to the given equations (16)-(20) over the interval $\left[0, t_{1}\right]$, and using the initial conditions $u_{l}{ }^{(K)}(0)=d_{K}$, the following approximate solution denoted by $u_{I}{ }^{(K)}(t)$ is obtained: 


$$
\mathrm{u}_{1}(\mathrm{~K})(\mathrm{t})=\sum_{k=0}^{K} U(k) t^{k}, \quad t \in\left[0, t_{1}\right]
$$

For $i \geq 1$, we use at each subinterval $\left[t_{i-1}, t_{i}\right]$ the initial conditions $u_{i}(K)\left(t_{i-1}\right)=u_{i-1}(K)\left(t_{i}-1\right)$ and apply the DTM to the given ODE over the subinterval $\left[t_{i-1}, t_{i}\right]$, where $x_{0}$ in Equation (13) is replaced by $t_{i-1}$. The process is repeated and generates a sequence of approximate solutions $u_{i}(t), i=1,2, \ldots, M$ for the solution:

$$
\mathrm{u}_{i}^{(\mathrm{K})}(\mathrm{t})=\sum_{k=0}^{K} U(k)\left(t-t_{i-1}\right)^{k}, \quad t \in\left[t_{i-1}, t_{i}\right] .
$$

Hence, the MsDTM assumes the following solution denoted by $u(K, M)$ [25].

$$
\mathrm{U}(\mathrm{K}, \mathrm{M})=\left\{\begin{array}{c}
\mathrm{u}_{1}^{(\mathrm{K})}(\mathrm{t}) t \in\left[0, t_{1}\right] \\
\mathrm{u}_{2}^{(\mathrm{K})}(\mathrm{t}) t \in\left[t_{1}, t_{2}\right] \\
\vdots \\
\mathrm{u}_{M}^{(\mathrm{K})}(t) t \in\left[t_{M-1}, t_{M}\right]
\end{array} .\right.
$$

Therefore, $S_{h}(t)$ can be expressed in terms of $s_{h}(t)$ given for different time intervals as :

$$
\begin{aligned}
& \mathrm{s}_{\mathrm{h}}(0, \mathrm{t})=0.99-0.000989 t-0.00008751605 t^{2}+\left(4.8357688 \times 10^{-7}\right) t^{3}-\left(1.4188247 \times 10^{-7}\right) t^{4}+ \\
& \left(3.9939127 \times 10^{-9}\right) t^{5}-\left(1.5241315 \times 10^{-10}\right) t^{6}, \\
& \mathrm{~s}_{\mathrm{h}}(1, \mathrm{t})=0.98892383-0.0011631298(-1+t)-0.00008687887(-1+t)^{2}-(4.6910337 \times \\
& \left.10^{-8}\right)(-1+t)^{3}-\left(1.2404874 \times 10^{-7}\right)(-1+t)^{4}+\left(3.1682438 \times 10^{-9}\right)(-1+t)^{5}-(1.237265 \times \\
& \left.10^{-10}\right)(-1+t)^{6}, \\
& \mathrm{~s}_{\mathrm{h}}(2, \mathrm{t})=0.98767365-0.0013375094(-2+t)-0.000087733991(-2+t)^{2}-(5.1377197 \times \\
& \left.10^{-7}\right)(-2+t)^{3}-\left(1.0993913 \times 10^{-7}\right)(-2+t)^{4}+\left(2.4993325 \times 10^{-9}\right)(-2+t)^{5}-(9.997816 \times \\
& \left.10^{-11}\right)(-2+t)^{6}, \\
& \mathrm{~s}_{\mathrm{h}}(3, \mathrm{t})=0.98624779-0.0015149465(-3+t)-0.000089911385(-3+t)^{2}-(9.3043039 \times \\
& \left.10^{-7}\right)(-3+t)^{3}-\left(9.8838704 \times 10^{-8}\right)(-3+t)^{4}+\left(1.9606311 \times 10^{-9}\right)(-3+t)^{5}-(8.0173043 \times \\
& \left.10^{-11}\right)(-3+t)^{6}, \\
& \mathrm{~s}_{\mathrm{h}}(4, \mathrm{t})=0.9846419-0.0016979466(-4+t)-0.000093277252(-4+t)^{2}-(1.3076947 \times \\
& \left.10^{-6}\right)(-4+t)^{3}-\left(9.0151256 \times 10^{-8}\right)(-4+t)^{4}+\left(1.5310186 \times 10^{-9}\right)(-4+t)^{5}-(6.3491359 \times \\
& \left.10^{-11}\right)(-4+t)^{6}, \\
& \mathrm{~s}_{\mathrm{h}}(5, \mathrm{t})=0.98284928-0.0018887775(-5+t)-0.000097726841(-5+t)^{2}-(1.654185 \times \\
& \left.10^{-6}\right)(-5+t)^{3}-\left(8.337465 \times 10^{-8}\right)(-5+t)^{4}+\left(1.1938498 \times 10^{-9}\right)(-5+t)^{5}-(4.925603 \times \\
& \left.10^{-11}\right)(-5+t)^{6}, \\
& \mathrm{~s}_{\mathrm{h}}(6, \mathrm{t})=0.98086104-0.0020895215(-6+t)-0.00010317841(-6+t)^{2}-(1.9766659 \times \\
& \left.10^{-6}\right)(-6+t)^{3}-\left(7.8080428 \times 10^{-8}\right)(-6+t)^{4}+\left(9.3618048 \times 10^{-10}\right)(-6+t)^{5}-(3.6906773 \times \\
& \left.10^{-11}\right)(-6+t)^{6} .
\end{aligned}
$$

Similarly, we can find solutions for $i_{h}(t), r_{h}(t), s_{v}(t)$ and $i_{v}(t)$ as a function of time (Appendix)

\section{Numerical Results and Discussion}

In this section, we plot the graph of the growth of $S_{h}(t)$ susceptible humans, $I_{h}(t)$ infected humans, $R_{h}(t)$ recovered humans, $S_{v}(t)$ susceptible vectors and $I_{v}(t)$ infected vectors using DTM, MsDTM (with step size 6) and RungeKutta method of order 4 implementing though Wolfram Mathematica 11. In Fig. 2, we compare the graphs of the susceptible humans using the three methods under consideration. It is found that the results of all three methods coincide for small $\mathrm{t}$ (in days) while after $\mathrm{t}=25$, the deviation increases in DTM as compared to MsDTM and RK4. deviation increases in DTM as compared to MsDTM and RK4. 

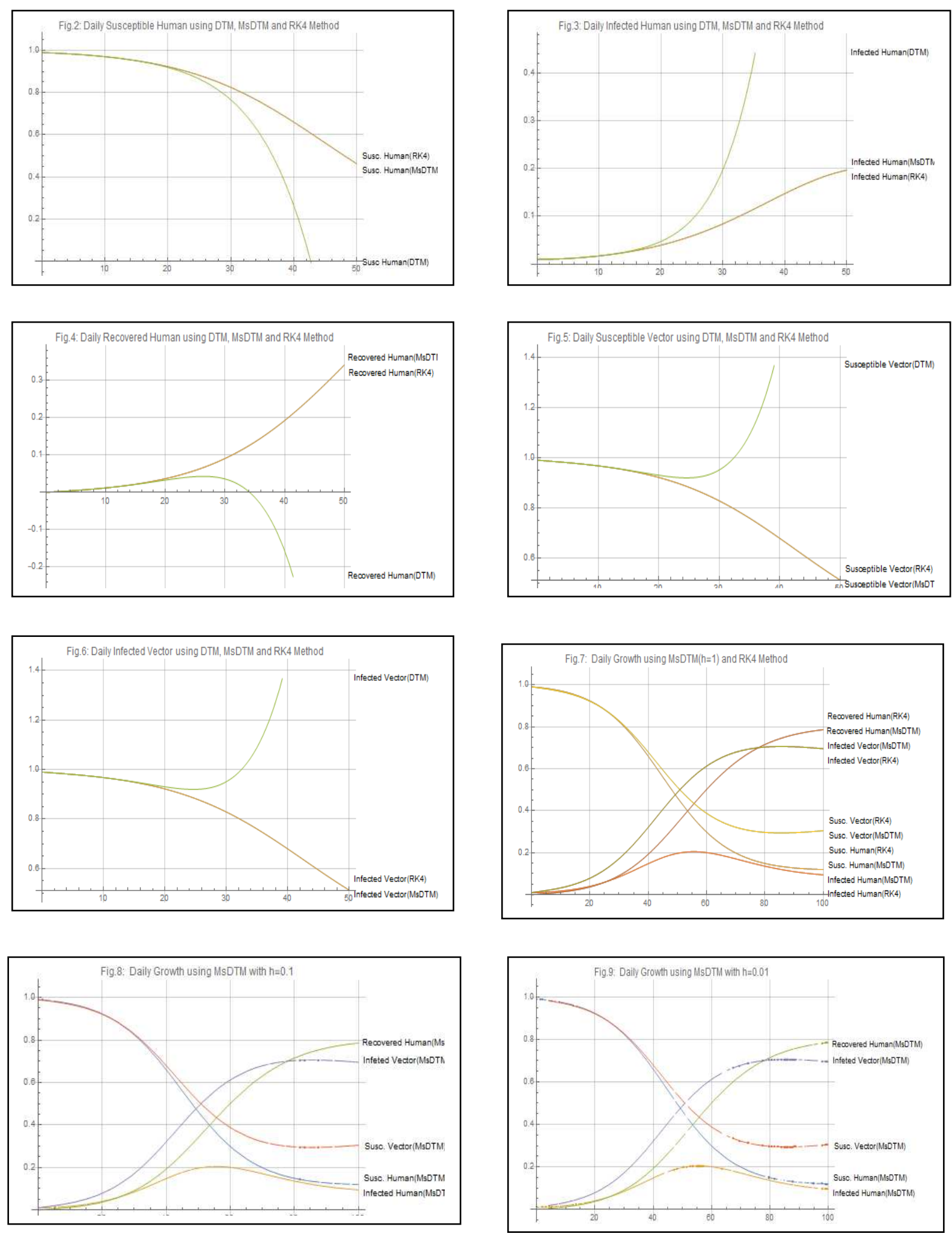
From the graphs of infected humans, recovered humans, susceptible vectors and infected vectors (Fig 3-6), it is observed that the solutions of DTM deviate after $t=25$, while there is no difference between the results of MsDTM (order of polynomial in terms of $t$ is 6) and RK4. The values computed using DTM deviates from the values obtained using MsDTM $(h=1)$ and RK4 significantly after $t=25$. Also, the values obtained for susceptible humans (and infected human, recovered humans, susceptible vectors and infected vectors) even for large time $(\mathrm{t}=100)$ is the same for MSDTM and RK4 (Fig. 7).

The MsDTM method has been further tested with the step size $h=0.1$ and 0.01 and is found to be consistent for all the solutions of infected humans, recovered humans, susceptible vectors and infected vectors(Fig. 7-9).

The values of susceptible humans for different time $t$ are tabulated here.

\begin{tabular}{|l|l|l|l|}
\hline \multicolumn{4}{|l|}{ Table 1 } \\
\hline Time & Values of $S_{h}(t)$ using DTM & Values of $S_{h}(t)$ using $M s D T M$ & Values of $S_{h}(t)$ using $R K 4$ \\
\hline 1 & 0.988924 & 0.988924 & 0.988924 \\
\hline 2 & 0.987674 & 0.987674 & 0.987674 \\
\hline 3 & 0.986248 & 0.986248 & 0.986248 \\
\hline 4 & 0.984642 & 0.984642 & 0.984642 \\
\hline 5 & 0.982849 & 0.982849 & 0.982849 \\
\hline 6 & 0.980861 & 0.980861 & 0.980861 \\
\hline 7 & 0.978666 & 0.978666 & 0.978666 \\
\hline 8 & 0.976252, & 0.976252 & 0.976253 \\
\hline 9 & 0.973604 & 0.973604 & 0.973605 \\
\hline 10 & 0.970704 & 0.970706 & 0.970707 \\
\hline 15 & 0.951709 & 0.951787 & 0.951788 \\
\hline 20 & 0.922373 & 0.923337 & 0.923337 \\
\hline 25 & 0.875308 & 0.881996 & 0.881995 \\
\hline 30 & 0.792391 & 0.824621 & 0.82462 \\
\hline 40 & 0.283974 & 0.659614 & 0.659614 \\
\hline 50 & -1.99193, & 0.461903 & 0.461904 \\
\hline 100 & -769.749 & 0.119838 & 0.119838 \\
\hline
\end{tabular}

\section{Conclusion}

The compartmental model is used to formulate and investigate dengue fever disease dynamics in a population with limited immunity. Two sets of dependent first-order non-linear equations for humans and vectors are obtained using the SIR model. The differential transform method (DTM), multi-step differential transform method (MsDTM) with step-size and RK4 using Mathematica is employed to obtain the semi-analytic solution in the form of time. The numerical simulations were carried out with the above stated three methods to determine the long term behaviour of susceptible, infected and recovered humans along with susceptible and infected vectors and displayed graphically for comparison. The solutions obtained using DTM is found to be the same as MsDTM and RK4 method for small-time $t(t<25)$. While, for $t>25$, the solutions obtained using MsDTM and RK4 are found to be the same. The MsDTM is an excellent alternative method for finding analytic solutions of non-linear dependent differential equations with the same numerical accuracy as obtained by the RK4 method.

\section{Bibliography}

[1] Abraham J. Arenas, Gilberto González-Parra, Benito M. Chen-Charpentier. "Dynamical analysis of the transmission of seasonal diseases using the differential transformation method". Mathematical and Computer Modelling 50.5-6 (2009): 765-776.

[2] Ahmad, Muhammad Zaini \& Alsarayreh, D. \& Alsarayreh, A. \& Qaralleh, Izzat. "Differential Transformation Method (DTM) for Solving SIS and SI Epidemic Models." Sains Malaysiana 46.10 (2017): 2007-2017.

[3] Akinboro, F.s \& Alao, S. \& Akinpelu, Folake. "Numerical Solution of SIR Model using the Differential Transformation Method and Variational Iteration Method." General Mathematics Notes 22.2 (2014): 82-92.

[4] Atika Radid, Karim Rhofir. "Partitioning differential transformation method to a SIR epidemic model under vaccination strategy." Int. J. of Ad. Applied. Math and Mech. 7.1 (2019): 9 - 19.

[5] Brahim Benhammouda, Hector Vazquez-Leal, Luis Hernandez-Martinez. "Modified Differential Transform Method for Solving the Model of Pollution for a System of Lakes." Discrete Dynamics in Nature and Society 2014 (2014): 12.

[6] Capasso, Vincenzo and Gabriella Serio. "A generalization of the Kermack-Mckendrick deterministic epidemic model." Mathematical Biosciences 42.1-2 (1978): 43-61. 
[7] Dengue:

https:/www.who.int/immunization/diseases/de ngue/en/.

[8] Dengue-Facts: https://www.who.int/newsroom/fact-sheets/detail/dengue-and-severedengue.

[9] Derouich M., Boutayeb A. "Dengue fever: Mathematical modelling and computer simulation." Applied Mathematics and Computation 177.2 (2006): 528-544.

[10] Derrick, N. R \& Grossman, S. L (1976). Differential Equation with application. Addison Wesley Publishing Company, Inc. Philippines

[11] Derrick, W R and Driessche, P Van Den. "A disease transmission model in a non-constant population.” Journal of Mathematical Biology 31.5 (1993): 495-512.

[12] Felix Yakubu Eguda, Andrawus James, Sunday Babuba. "The Solution of a Mathematical Model for Dengue Fever Transmission Using Differential Transformation Method." Journal of Nigerian Society of Physical Sciences (2019): 82-87.

[13] Gupta, B. Dhar and P. K. "Numerical Solution of Tumor-Immune Model with Targeted Chemotherapy by Multi-Step Differential Transformation Method." Ed. Balas V., Esposito A., Gope S. Dawn S. Springer, Cham, 2020. 404-411.

[14] Hethcote H.W., Levin S.A. "Periodicity in epidemiological models." Gross L., Hallam T.G., Levin S.A. Applied Mathematical Ecology. Berlin: Springer-Verlag, 1989. 193.

[15] Hethcote Herbert W, Driessche P Van Den. "Some epidemiological models with nonlinear incidence." Journal of Mathematical Biology 29.3 (1997): 271-287.

[16] Hethcote, H. "The Mathematics of Infectious Diseases." SIAM Review 42.4 (2000): 599653.

[17] Ibrahim, S F M and Ismai, S M 1. "A New Modification of the Differential Transform Method for a SIRC Influenza Model." International Journal of Computer Applications 16.19 (2013): 1-15.

[18] K. A. Gepreel, A. M. S. Mahdy, M. S. Mohamed, A. Al-Amiri. "Reduced Differential Transform Method for Solving Nonlinear Biomathematics Models." Computers, Materials \& Continua 61.3 (2019): 979-994.

[19] Kermack, W. O. and Mckendrick, A. G. "Contribution to the Mathematical Theory of Epidemics (Part 1)." Proc. R. Soc. Lond. B. Biol. Sci. 138 (1932): 55-83.

[20] Kermack, W. O. and Mckendrick, A. G. "Contribution to the Mathematical Theory of Epidemics (Part II).” Proc. R. Soc. Lond. B. Biol. Sci. 141 (1932): 94-112.

[21] Kermack, W. O., McKendrick, A. G. "A contribution to the mathematical theory of epidemics." Proceedings of the Royal Society of London A: Mathematical, physical and engineering science 115 (1927): 700-721.

[22] Keskin G., Oturanc Y. "Reduced Differential Transform Method for Solving Linear and NonLinear Wave Equations." Iranian Journal of Science \& Technology, Transaction A 34.A2 (2010): 113-122.

[23] Meksianis Z. Ndii, Nursanti Anggriani, and Asep K. Supriatna. "Application of differential transformation method for solving dengue transmission mathematical model." AIP Conference Proceedings. 2018.

[24] Mohammad Mehdi Rashidi, Ali J. Chamkha, Mohammad Keimanesh. "Application of MultiStep Differential Transform Method on Flow of a Second-Grade Fluid over a Stretching or Shrinking Sheet." American Journal of Computational Mathematics 6 (2011): 119-128.

[25] Munganga, Justin \& Mwambakana, Jeanine \& Maritz, Riette \& Batubenge, Tshidibi \& Moremedi, Marcia. "Introduction of the differential transform method to solve differential equations at the undergraduate level." International Journal of Mathematical Education in Science and Technology 5 (2014).

[26] Peter, Olumuyiwa \& Ibrahim, Mohammed Olanrewaju. "Application of Differential Transform Method in Solving a Typhoid Fever Model." International Journal of Mathematical Analysis And Optimization: Theory And Applications 1 (2017): 250-260.

[27] Peter, Olumuyiwa \& Oluwaseun, Akinduko \& Christie, Ishola \& Afolabi, Ahmed \& Ganiyu, Afees. "Series Solution of Typhoid Fever Model Using Differential Transform Method." Malaysian Journal of Computing 3.1 (2018):6780.

[28] Sharaf Mohmoud, Mohamed Gubara. "Reduced Differential Transform Method for Solving Linear and Nonlinear Goursat." Applied Mathematics 7 (2016): 1049-1056.

[29] Stanaway, Jeffrey D, et al. "The global burden of dengue: an analysis from the Global Burden of Disease Study 2013." The Lancet Infectious Diseases 16.6 (2016): 712-723.

[30] Taghavi A., Babaei A., Mohammadpour A. "Application of Reduced Differential Transform Method for Solving Nonlinear Reaction-Diffusion-Convection Problems." Applications and Applied Mathematics: An International Journal 10.1 (2015): 162-170.

[31] Tuteja, Gurpreet Singh. "A Study of Epidemic Model of Dengue with limited Immunity based on SIR model." International Journal of Research and Analytical Reviews 5.1 (2018): 589-595.

[32] Zaid, M. Odibat "Differential transform method for solving Volterra integral equation 
with separable kernels." Mathematical and Computer Modelling 48.7-8 (2008).

[33] Zaid, M. Odibat Cyrille Bertelle, M.A. AzizAlaoui, Gérard H.E. Duchamp. "A multi-step differential transform method and application to non-chaotic or chaotic systems." Computers \& Mathematics with Applications 59.4 (2010): 1462-1472.

[34] Zhou, J K. Differential Transformation and Its Applications for Electrical Circuits. Wuhan, China: Huazhong University Press, 1986.

\section{Appendix}

$I_{h}(t)$ can be expressed in terms of $i_{h}(t)$ given for different time intervals as :

$i_{\mathrm{h}}(0, \mathrm{t})=0.01-0.000011 t+$ $0.00009311605 t^{2}-\left(3.6062986 \times 10^{-6}\right) t^{3}+$ $\left(2.3992481 \times 10^{-7}\right) t^{4}-(8.9904544 \times$ $\left.10^{-9}\right) t^{5}+\left(3.106649 \times 10^{-10}\right) t^{6}$

$i_{\mathrm{h}}(1, \mathrm{t})=0.010078741+0.00016532975(-1+$ $t)+0.000083651272(-1+t)^{2}-(2.7305992 \times$ $\left.10^{-6}\right)(-1+t)^{3}+\left(1.9932694 \times 10^{-7}\right)(-1+$ $t)^{4}-\left(7.3068445 \times 10^{-9}\right)(-1+t)^{5}+$ $\left(2.5247388 \times 10^{-10}\right)(-1+t)^{6}$,

$i_{\mathrm{h}}(2, \mathrm{t})=0.010325184+0.00032520273(-2+$ $t)+0.000076586003(-2+t)^{2}-(2.0015632 \times$ $\left.10^{-6}\right)(-2+t)^{3}+\left(1.6632944 \times 10^{-7}\right)(-2+$ $t)^{4}-\left(5.9398296 \times 10^{-9}\right)(-2+t)^{5}+$ $\left(2.0476682 \times 10^{-10}\right)(-2+t)^{6}$,

$i_{\mathrm{h}}(3, \mathrm{t})=0.010725131+0.00047300685(-3+$ $t)+0.000071522838(-3+t)^{2}-(1.3917561 \times$ $\left.10^{-6}\right)(-3+t)^{3}+\left(1.3949613 \times 10^{-7}\right)(-3+$ $t)^{4}-\left(4.8326816 \times 10^{-9}\right)(-3+t)^{5}+$ $\left(1.6555236 \times 10^{-10}\right)(-3+t)^{6}$,

$i_{\mathrm{h}}(4, \mathrm{t})=0.011268404+0.00061241204(-4+$ $t)+0.000068138599(-4+t)^{2}-(8.7895844 \times$ $\left.10^{-7}\right)(-4+t)^{3}+\left(1.1764652 \times 10^{-7}\right)(-4+$ $t)^{4}-\left(3.9394893 \times 10^{-9}\right)(-4+t)^{5}+$ $\left(1.3320377 \times 10^{-10}\right)(-4+t)^{6}$,

$i_{\mathrm{h}}(5, \mathrm{t})=.01194819+0.00074650402(-5+$ $t)+0.000066170121(-5+t)^{2}-(4.4524478 \times$ $\left.10^{-7}\right)(-5+t)^{3}+\left(9.980683 \times 10^{-8}\right)(-5+$ $t)^{4}-\left(3.2231794 \times 10^{-9}\right)(-5+t)^{5}+$ $\left(1.0639173 \times 10^{-10}\right)(-5+t)^{6}$,

$i_{\mathrm{h}}(6, \mathrm{t})=0.012760515+0.00087789225(-6+$ $t)+0.000065402521(-6+t)^{2}-(7.6239267 \times$ $\left.10^{-8}\right)(-6+t)^{3}+\left(8.5169996 \times 10^{-8}\right)(-6+$ $t)^{4}-\left(2.6539013 \times 10^{-9}\right)(-6+t)^{5}+$ $\left(8.4029958 \times 10^{-11}\right)(-6+t)^{6}$.

$R_{h}(t)$ can be expressed in terms of $r_{h}(t)$ given for different time intervals as :

$r_{h}(0, \mathrm{t})=0.001 t-\left(5.6 \times 10^{-6}\right) t^{2}+$

$\left(3.1227217 \times 10^{-6}\right) t^{3}-(9.8042336 \times$ $\left.10^{-8}\right) t^{4}+\left(4.9965416 \times 10^{-9}\right) t^{5}-$ $\left(1.5825175 \times 10^{-10}\right) t^{6}$, $r_{h}(1, \mathrm{t})=0.00099742952+$ $0.00099780006(-1+t)+(3.2275972 \times$ $\left.10^{-6}\right)(-1+t)^{2}+\left(2.7775095 \times 10^{-6}\right)(-1+$ $t)^{3}-\left(7.5278191 \times 10^{-8}\right)(-1+t)^{4}+$ $\left(4.1386007 \times 10^{-9}\right)(-1+t)^{5}-(1.2874739 \times$ $\left.10^{-10}\right)(-1+t)^{6}$,

$r_{h}(2, \mathrm{t})=0.0020011634+0.0010123066(-2+$ $t)+0.000011147988(-2+t)^{2}+(2.5153352 \times$ $\left.10^{-6}\right)(-2+t)^{3}-\left(5.6390302 \times 10^{-8}\right)(-2+$ $t)^{4}+\left(3.4404971 \times 10^{-9}\right)(-2+t)^{5}-$

$\left(1.0478866 \times 10^{-10}\right)(-2+t)^{6}$, $r_{h}(3, \mathrm{t})=0.0030270803+0.0010419396(-3+$ $t)+0.000018388548(-3+t)^{2}+(2.3221865 \times$ $\left.10^{-6}\right)(-3+t)^{3}-\left(4.0657423 \times 10^{-8}\right)(-3+$ $t)^{4}+\left(2.8720505 \times 10^{-9}\right)(-3+t)^{5}-$

$\left(8.5379312 \times 10^{-11}\right)(-3+t)^{6}$,

$r_{h}(4, \mathrm{t})=0.0040896928+0.0010855345(-4+$ $t)+0.000025138653(-4+t)^{2}+(2.1866532 \times$ $\left.10^{-6}\right)(-4+t)^{3}-\left(2.749526 \times 10^{-8}\right)(-4+$ $t)^{4}+\left(2.4084707 \times 10^{-9}\right)(-4+t)^{5}-$

$\left(6.9712415 \times 10^{-11}\right)(-4+t)^{6}$, $r_{h}(5, \mathrm{t})=.0052025275+0.0011422734(-5+$ $t)+0.00003155672(-5+t)^{2}+(2.0994297 \times$ $\left.10^{-6}\right)(-5+t)^{3}-\left(1.643218 \times 10^{-8}\right)(-5+$ $t)^{4}+\left(2.0293296 \times 10^{-9}\right)(-5+t)^{5}-$

$\left(5.7135696 \times 10^{-11}\right)(-5+t)^{6}$,

$r_{h}(6, \mathrm{t})=0.0063784426+0.0012116293(-6+$ $t)+0.000037775885(-6+t)^{2}+(2.0529052 \times$ $\left.10^{-6}\right)(-6+t)^{3}-\left(7.0895673 \times 10^{-9}\right)(-6+$ $t)^{4}+\left(1.7177208 \times 10^{-9}\right)(-6+t)^{5}-$

$\left(4.7123185 \times 10^{-11}\right)(-6+t)^{6}$.

$S_{v}(t)$ can be expressed in terms of $s_{h}(t)$ given for different time intervals as :

$\mathrm{s}_{\mathrm{v}}(0, \mathrm{t})=0.99-0.00188 \mathrm{t}+0.000012369 t^{2}-$ $\left(6.196514 \times 10^{-6}\right) t^{3}+\left(2.0586103 \times 10^{-7}\right) t^{4}-$ $\left(1.0315079 \times 10^{-8}\right) t^{5}+\left(3.5314596 \times 10^{-10}\right) t^{6}$, $s_{v}(1, \mathrm{t})=.98812637-0.0018730776(-1+t)-$ $\left(5.083454 \times 10^{-6}\right)(-1+t)^{2}-(5.4695297 \times$ $\left.10^{-6}\right)(-1+t)^{3}+\left(1.5921526 \times 10^{-7}\right)(-1+$ $t)^{4}-\left(8.4124306 \times 10^{-9}\right)(-1+t)^{5}+$

$\left(2.838726 \times 10^{-10}\right)(-1+t)^{6}$,

$s_{v}(2, \mathrm{t})=0.98624289-0.0018990567(-2+$ $t)-0.000020616794(-2+t)^{2}-(4.9114073 \times$ $\left.10^{-6}\right)(-2+t)^{3}+\left(1.2112274 \times 10^{-7}\right)(-2+$ $t)^{4}-\left(6.8789158 \times 10^{-9}\right)(-2+t)^{5}+$ $\left(2.2948151 \times 10^{-10}\right)(-2+t)^{6}$,

$s_{v}(3, \mathrm{t})=0.98431842-0.0019545731(-3+$ t) $-0.000034689765(-3+t)^{2}-(4.4913453 \times$ $\left.10^{-6}\right)(-3+t)^{3}+\left(8.994369 \times 10^{-8}\right)(-3+$ $t)^{4}-\left(5.6354076 \times 10^{-9}\right)(-3+t)^{5}+$ $\left(1.8673913 \times 10^{-10}\right)(-3+t)^{6}$, $s_{v}(4, \mathrm{t})=0.98232475-0.002037094(-4+t)-$ $0.000047677801(-4+t)^{2}-(4.1843699 \times$ $\left.10^{-6}\right)(-4+t)^{3}+\left(6.4389774 \times 10^{-8}\right)(-4+$ $t)^{4}-\left(4.6196563 \times 10^{-9}\right)(-4+t)^{5}+$ $\left(1.5321104 \times 10^{-10}\right)(-4+t)^{6}$,

$S_{v}(5, \mathrm{t})=0.98023585-0.0021447673(-5+$ $t)-0.000059888555(-5+t)^{2}-(3.970084 \times$ $\left.10^{-6}\right)(-5+t)^{3}+\left(4.3450549 \times 10^{-8}\right)(-5+$ $t)^{4}-\left(3.7821738 \times 10^{-9}\right)(-5+t)^{5}+$

$\left(1.2704791 \times 10^{-10}\right)(-5+t)^{6}$,

$s_{v}(6, \mathrm{t})=0.97802727-0.002276299(-6+t)-$ $0.000071574086(-6+t)^{2}-(3.8316718 \times$ 
$\left.10^{-6}\right)(-6+t)^{3}+\left(2.633759 \times 10^{-8}\right)(-6+$ $t)^{4}-\left(3.0832065 \times 10^{-9}\right)(-6+t)^{5}+$ $\left(1.0683288 \times 10^{-10}\right)(-6+t)^{6}$.

$I_{v}(t)$ can be expressed in terms of $i_{v}(t)$ given for different time intervals as :

$i_{\mathrm{v}}(0, \mathrm{t})=0.01+0.00188 t-0.000012369 t^{2}+$ $\left(6.196514 \times 10^{-6}\right) t^{3}-\left(2.0586103 \times 10^{-7}\right) t^{4}+$ $\left(1.0315079 \times 10^{-8}\right) t^{5}-\left(3.5314596 \times 10^{-10}\right) t^{6}$, $i_{\mathrm{v}}(1, \mathrm{t})=0.011873632+0.0018730776(-1+$ $t)+\left(5.083454 \times 10^{-6}\right)(-1+t)^{2}+$ $\left(5.4695297 \times 10^{-6}\right)(-1+t)^{3}-(1.5921526 \times$ $\left.10^{-7}\right)(-1+t)^{4}+\left(8.4124306 \times 10^{-9}\right)(-1+$ $t)^{5}-\left(2.838726 \times 10^{-10}\right)(-1+t)^{6}$,

$i_{\mathrm{v}}(2, \mathrm{t})=.013757111+0.0018990567(-2+$ $t)+0.000020616794(-2+t)^{2}+(4.9114073 \times$ $\left.10^{-6}\right)(-2+t)^{3}-\left(1.2112274 \times 10^{-7}\right)(-2+$ $t)^{4}+\left(6.8789158 \times 10^{-9}\right)(-2+t)^{5}-$ $\left(2.2948151 \times 10^{-10}\right)(-2+t)^{6}$, $i_{\mathrm{v}}(3, \mathrm{t})=.015681582+0.0019545731(-3+$ $t)+0.000034689765(-3+t)^{2}+(4.4913453 \times$ $\left.10^{-6}\right)(-3+t)^{3}-\left(8.994369 \times 10^{-8}\right)(-3+$ $t)^{4}+\left(5.6354076 \times 10^{-9}\right)(-3+t)^{5}-$ $\left(1.8673913 \times 10^{-10}\right)(-3+t)^{6}$, $i_{\mathrm{v}}(4, \mathrm{t})=0.017675251+0.002037094(-4+$ $t)+0.000047677801(-4+t)^{2}+(4.1843699 \times$ $\left.10^{-6}\right)(-4+t)^{3}-\left(6.4389774 \times 10^{-8}\right)(-4+$ $t)^{4}+\left(4.6196563 \times 10^{-9}\right)(-4+t)^{5}-$ $\left(1.5321104 \times 10^{-10}\right)(-4+t)^{6}$, $i_{\mathrm{v}}(5, \mathrm{t})=0.019764147+0.0021447673(-5+$ $t)+0.000059888555(-5+t)^{2}+(3.970084 \times$ $\left.10^{-6}\right)(-5+t)^{3}-\left(4.3450549 \times 10^{-8}\right)(-5+$ $t)^{4}+\left(3.7821738 \times 10^{-9}\right)(-5+t)^{5}-$ $\left(1.2704791 \times 10^{-10}\right)(-5+t)^{6}, \quad i_{\mathrm{v}}(6, \mathrm{t})=$ $0.021972734+0.002276299(-6+t)+$ $0.000071574086(-6+t)^{2}+(3.8316718 \times$ $\left.10^{-6}\right)(-6+t)^{3}-\left(2.633759 \times 10^{-8}\right)(-6+$ $t)^{4}+\left(3.0832065 \times 10^{-9}\right)(-6+t)^{5}-$ $\left(1.0683288 \times 10^{-10}\right)(-6+t)^{6}$. 